\title{
Intrinsic oscillations of auditory networks in schizophrenia and bipolar disorder
}

\author{
Murat Ilhan Atagun ${ }^{*}{ }^{\circledR}$, Burak Akin ${ }^{2 \odot}$, Oktay Algin ${ }^{1,3,4}$, Elif Muazzez Sikoglu ${ }^{5}$, \\ Serdar Suleyman Can ${ }^{1,6}$, Semra Ulusoy Kaymak ${ }^{6 \odot}$, Ali Caykoylu' ${ }^{1,6}$, \\ Constance Moore $^{5,7}$, Mary Phillips ${ }^{8}{ }^{\circledR}$, Dost Ongur ${ }^{9,10 \odot}$ \\ ${ }^{1}$ Ankara Yildirim Beyazit University, Faculty of Medicine, Department of Psychiatry, Ankara - Turkey \\ ${ }^{2}$ University of Freiburg, Faculty of Medicine, Department of Radiology, Freiburg - Germany \\ ${ }^{3}$ Ankara Atatürk Training and Education Hospital, Department of Radiology, Ankara - Turkey \\ ${ }^{4}$ Bilkent University, National Magnetic Resonance Imaging Research Center, Department of Radiology, Ankara - Turkey \\ ${ }^{5}$ University of Massachusetts Medical School, Department of Psychiatry, Worcester, Massachusetts - USA \\ ${ }^{6}$ Ankara Atatürk Training and Education Hospital, Department of Psychiatry, Ankara - Turkey \\ 7University of Massachusetts Medical School, Department of Radiology, Worcester, Massachusetts - USA \\ ${ }^{8}$ University of Pittsburgh Medical School, Department of Psychiatry, Pittsburgh, Pennsylvania - USA \\ ${ }^{9}$ Harvard University Medical School, Department of Psychiatry, Boston, Massachusetts - USA \\ ${ }^{10}$ McLean Hospital, Psychotic Disorders Division, Department of Mental Health, Belmont, Massachusetts - USA
}

\begin{abstract}
Objective: Several types of evidence have shown that auditory networks are dysfunctional in schizophrenia (Sch) and bipolar disorders (BD). Auditory cortices show abnormalities in hallucinations or during remission. This study aimed to assess resting-state connectivity of auditory cortices in Sch and BD.

Method: Patients with BD-1 $(n=28), B D-2(n=21)$, Sch $(n=30)$, and healthy controls $(H C, n=30)$ were enrolled into the study. A 3 Tesla whole-body magnetic resonance imaging (MRI) system with a 32-channel phase-array head coil was used to acquire the MRI data. T1-weighted anatomical and gradient-echo based Echoplanar Imaging sequences were used. MATLAB and Freesurfer software packages were used for data analyses. Connectivity alterations within the auditory network guided our further seedbased connectivity analysis.

Results: The left angular gyrus volume was decreased in Sch and BD-2 groups. The supramarginal gyrus had hyperconnectivity with the medial prefrontal cortices and decreased connectivity with the medial superior temporal gyrus (STG) in the BD-1 and BD-2 groups. The left superior temporal sulcus (STS) had increased connectivity with the bilateral posterior cingulate cortex in BD-1 and BD-2 and increased connectivity with the dorsal prefrontal cortices in the Sch group. The STS had increased connectivity with the medial STG in the BD-1 and Sch groups, whereas connectivity decreased in the BD-2 group.
\end{abstract}

Conclusion: These findings suggest that functional connectivity of resting-state networks are altered in BD and Sch. Auditory network alterations may predispose to dysfunctional auditory information processing. Further studies are needed to determine the relationship between symptoms and auditory network dysfunction.

Keywords: Auditory cortex, bipolar disorder, network, schizophrenia.

How to cite this article: Atagun MI, Akin B, Algin O, Sikoglu EM, Can SS, Ulusoy-Kaymak S, Caykoylu A, Moore C, Philips M, Ongur D. Intrinsic Oscillations of Auditory Networks in Schizophrenia and Bipolar Disorder. Dusunen Adam The Journal of Psychiatry and Neurological Sciences 2019;32:4-13.

Correspondence: Murat Ilhan Atagun, Universities region, Ihsan Dogramaci Bulvari, No: 6, 06800, Bilkent, Cankaya, Ankara, Turkey Phone: +90-312-291-2525/3286 E-mail: miatagun@ybu.edu.tr

Received: July 04, 2018; Revised: October 23, 2018; Accepted: December 13, 2018 


\section{INTRODUCTION}

The developmental trajectory of the auditory cortices extends until late adolescence and is vulnerable to disruption $(1,2)$. Several lines of evidence have demonstrated that auditory cortices are among the most volatile brain regions in patients with schizophrenia and bipolar disorder. Pressured speech, flight of ideas, auditory hallucinations, and verbal memory deficits are the symptom domains related with the auditory cortices in schizophrenia and bipolar disorder (2). Several histopathological examinations (3-5) and neuroimaging studies (6-17) have consistently reported abnormalities in auditory cortices in schizophrenia and bipolar disorder.

The primary auditory cortex (PAC), Wernicke's Area (WA), the inferior parietal lobe (IPL), and Broca's Area (BA) and their anatomical connections constitute the auditory networks, notably including two central heteromodal association areas (i.e. Wernicke's and Broca's Areas) (18). Different units within the auditory cortices participate in processing different features of auditory input (19). Hence, the integration between the components of the auditory cortices by hardly-wired networks at diverse scales is crucial for such distributed and collaborative procedures of auditory perception. Two main pathways, the ventral and dorsal streams, are associated with semantic and spatial processing, respectively (20).

Resting-state functional connectivity is based on the correlations of spontaneous brain activity while the brain is not engaged in any task. Correlations of lowfrequency fluctuations are interpreted as evidence of intrinsic, functional connections between brain regions. As auditory hallucinations typically emerge at rest, it has been shown that the correlation of the brain activity shows that resting-state functional connectivity abnormalities of the auditory cortices are significantly higher in hallucinating patients, in comparison to nonhallucinating patients with schizophrenia $(21,22)$. Shinn et al. (23) have observed differences between schizophrenia patients with and without auditory hallucinations in resting-state functional connectivity of the left superior temporal gyrus. The authors reported that left superior temporal gyrus (STG) had hyperconnectivity with cortical regions of the forebrain that are involved in speech, memory formation, executive functions, and self-referential thoughts. On the other hand, abnormalities of the auditory cortices are not limited to auditory hallucinations in schizophrenia and bipolar disorder. Auditory emotional $(24,25)$ and cognitive (10-12) processing deficits are observed in schizophrenia and bipolar disorder. These findings may indicate a dysfunction in auditory networks even when the patients are in remission (10).

Independent component analysis (ICA)-based resting-state functional Magnetic Resonance Imaging (fMRI) studies have reported abnormal connectivity between auditory cortices and other brain regions in schizophrenia and bipolar disorder $(26,27)$. Voxel-wise functional connectivity analyses are the sum of ICAderived within- and between-network analyses (28). Preprocessing steps and statistical analyses differ between the methods. Voxel-wise correlation analysis is able to show correlation between time series of brain regions and the voxel of interest. Therefore, this study aimed to assess seed-based resting-state functional connectivity between auditory cortices and other brain regions in schizophrenia and bipolar disorder.

\section{METHOD}

This study was approved by the local Ethics Committee. All participants provided written informed consent before enrollment. Consecutive outpatients were invited to participate in the study and accepting patients were enrolled, including 30 (12 women) patients with schizophrenia, 28 (15 women) with bipolar I disorder (BD-1), 21 (12 women) with bipolar II disorder (BD-2) and 30 (17 women) healthy controls (HC). Diagnoses were checked by Structured Clinical Interview According to DSM-IV (SCID-1) (29). Young Mania Rating Scale (YMRS) (30,31), Hamilton Depression Rating Scale (HDRS) (32,33), Brief Psychiatric Rating Scale (BPRS) (34), Scale for the Assessment of Negative Symptoms (SANS) $(35,36)$, Scale for the Assessment of Positive Symptoms (SAPS) $(37,38)$, and Edinburgh Handedness Inventory (39) were the clinical evaluation tools (administered by MIA). MRI scans were performed immediately after the clinical assessments. All patients included in this study were clinically stable. Exclusion criteria were any history of brain surgery or major physical trauma, diabetes mellitus, hypertension, metallic implants that are not compatible with MRI sessions, hearing disabilities, and any psychiatric comorbidity or lifetime history of substance abuse. We enrolled patients regardless of psychotropic medications; only patients on benzodiazepines were excluded.

\section{Data Acquisition}

A 3 Tesla whole-body magnetic resonance imaging (MRI) system (Magnetom Tim Trio, Siemens AG, Erlangen Germany) with a 32-channel phase-array head coil was used to acquire the MR data at the 
UMRAM (National Magnetic Resonance Research Center, Ankara, Turkey). T1-weighted anatomical MRIs (MPRAGE sequence, 256x256 voxels, TR: 2000 msec, TE: $3.02 \mathrm{msec}$, FOV read: 215, FOV phase: 100, slice thickness: $0.84,192$ slices) were acquired for diagnostic and localization purposes. A gradient echobased EPI sequence was used to acquire functional MRI data (TR: $2000 \mathrm{msec}$, TE:40 msec, Flip Angle: $71^{\circ}$ and 3 $\mathrm{mm}$ isometric-voxel resolution, $64 \times 64 \times 32$ matrix size with 90 volumes). Patients were instructed to close their eyes but not to sleep during the scan.

\section{Data Analysis}

Brain extraction (FSL 5.0 BET) was performed on T1-weighted anatomical images and linear transformation matrices for their individual functional spaces were calculated. Automatic cortical parcellation was performed using Freesurfer v5.3.0 (40) and 14 auditory regions of interest (ROIs) defined previously were labeled for each subject (Figure 1a). The first two volumes of functional data were discarded and slice-scan time correction was performed. Motion correction was done by using rigid body linear registration (FSL 5.0 MCFLIRT). A band-pass filter was applied to restrict signal variations in between 0.01 and $0.1 \mathrm{~Hz}(41)$. Auditory regions in anatomical space registered to individual functional spaces. Mean time courses were extracted for each of the 14 previously labeled ROIs. Pairwise functional connectivity changes of 14 ROIs were investigated. Within-auditory network analysis revealed connectivity changes in SMG and primary auditory cortices (Table 3). The ROIs with significant pairwise connectivity changes were used as seeds (Figure 1a) and a whole-brain connectivity map with these seed ROIs was further investigated. The connectivity of the seeds (ROI 9, 13 and 14) that had significant whole-brain connectivity differences between the groups is presented in Figure 2.

\section{Statistical Analysis}

All processing routines were performed using MATLAB R2016b. One-way ANOVA was conducted on pairwise correlation values to assess functional group differences. Functional data was smoothed with Gaussian kernel (FWHM $=7 \mathrm{~mm}$ ); then whole-brain connectivity maps

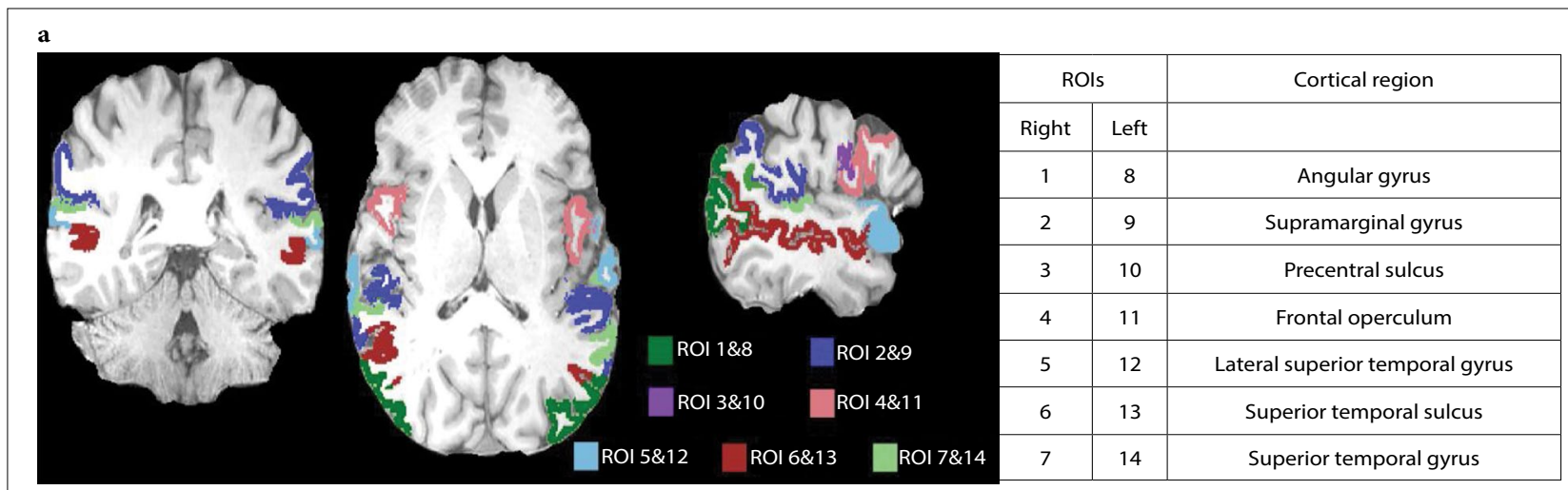

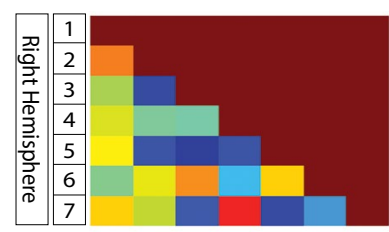
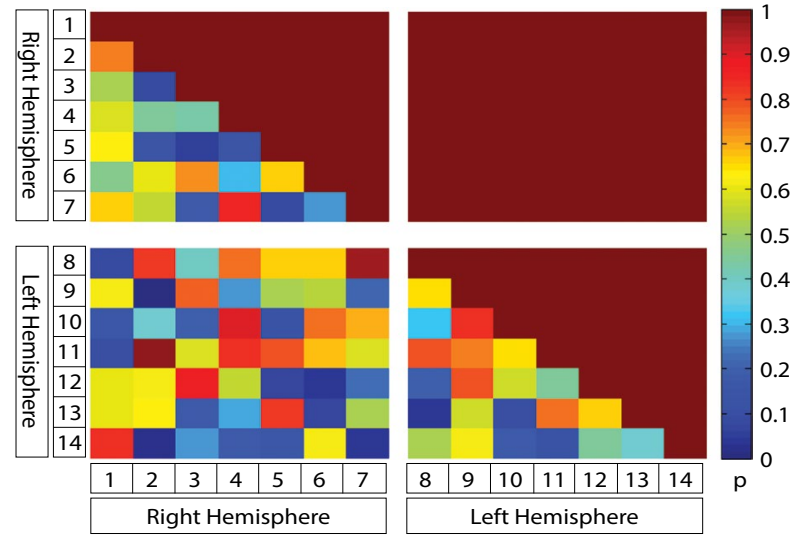

c
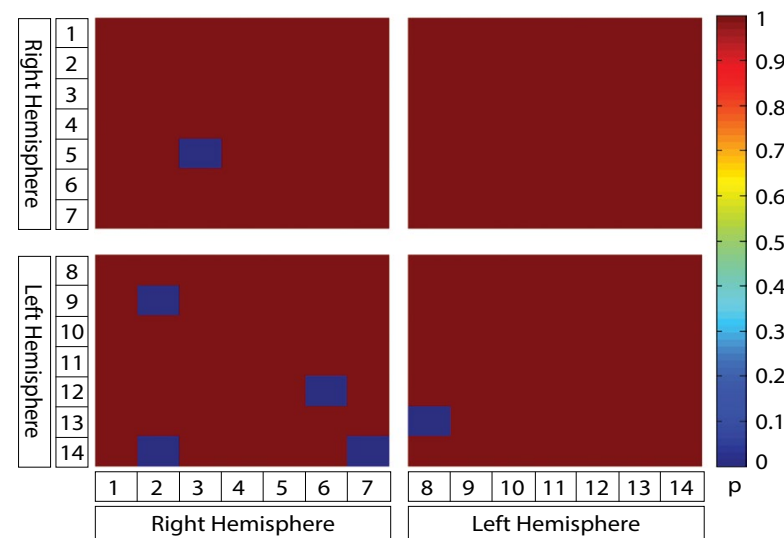

Figure 1: Brain regions of interest demonstrated in one subject (a), One-way ANOVA results of pairwise correlations (b). p $<0.05$ thresholded table (c). Four separate subplots show inter-hemispheric pairwise correlations (between left and right hemispheres) and intra-hemispheric (between left and left, right and right) correlations. Color bars represent $\mathrm{p}$ values. 
were calculated using those regions as a seed (42). T-test with FDR $(\mathrm{q} \leq 0.05)$ correction was used to get seedbased connectivity maps. Statistical analyses of the volumes were performed separately. Other statistical analyses were performed with SPSS 22 (IBM Corp., Armonk, NY). Distribution characteristics of the data

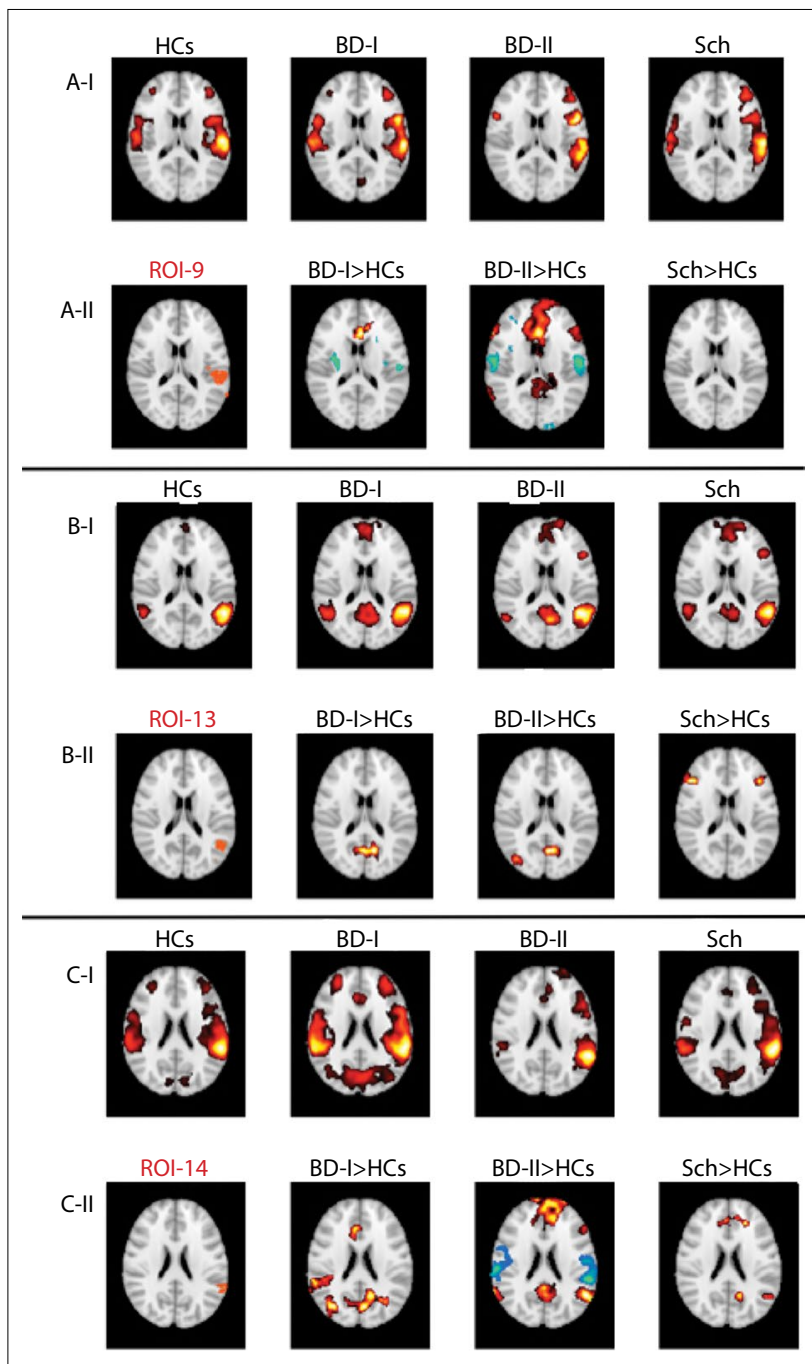

Figure 2: Group-level connectivity maps by using (A) left supramarginal gyrus (ROI-9; Brodmann 40), (B) medial left superior temporal sulcus (ROI-13; Brodmann 41, 42) and (C) lateral left superior temporal gyrus (ROI-14; Brodmann 21, 22) as seeds. Color bars represent Pearson Correlation values $(r>0.5)$. Connectivity maps of each group are presented in A-I, B-I, and C-I. Significant connectivity differences between groups are presented in A-II, B-II and C-II. Changes of connectivity values are presented as increase (red-yellow) and decrease (blue-green) with $t$ scores $[|t|>2]$ in comparison to the HC group. HCs: Healthy Controls, BD-I: Bipolar I Disorder, BD-II: Bipolar II Disorder, Sch: Schizophrenia. There were significant connectivity differences between the groups at ROI-9 (A-II: BD-I vs. HC, BD-II vs. HC), ROI-13 (B-II: BD-I vs. HC, BD-II vs. HCs, Sch vs. HCs) and ROI-14 (C-II: BD-I vs. HCs, BD-II vs. HCs, Sch vs. HCs). were checked with Kolmogorov-Smirnov and ShapiroWilk tests. Variables with Gaussian distribution were compared with $t$ test and one-way ANOVA. Categorical variables were compared with chi-square test. Homogeneity of variances was checked with Levene's homogeneity of variance test for post-hoc tests. Tukey test was performed for variables with homogeneous variances, whereas Tamhane test was performed for non-homogeneous variances. The level of statistical significance for $\mathrm{p}$ values was 0.05 .

\section{RESULTS}

Sociodemographic and clinical characteristics of the patient groups are presented in Table 1. There were no significant age and sex differences between patient groups and healthy subjects. BPRS scores differed between the groups: The schizophrenia group had a significantly higher score than the bipolar I disorder $(B D-1)(\mathrm{p}<0.001)$ and bipolar II disorder $(\mathrm{BD}-2)(\mathrm{p}<0.001)$ groups.

ANOVA showed that there were volume differences between groups at the following locations: right hemisphere lateral STG (ROI-5), left angular gyrus (ROI8), and left STG (ROI-14) (Table 2). Post-hoc comparison showed that the mean volume of the left angular gyrus in the schizophrenia and bipolar II disorder groups was significantly smaller than in healthy controls. The left STG in the schizophrenia group was significantly smaller than in the healthy control group. The difference between the groups in the lateral part of the STG in the right hemisphere (ROI-5) was at trend level in post-hoc comparison (schizophrenia $<$ healthy controls, $\mathrm{p}=0.0578$; schizophrenia $<$ bipolar 1 disorder, $\mathrm{p}=0.061$ ).

Pairwise correlations between seed regions showed that the most significant functional connectivity changes are at SMG (ROI-2 and ROI-9) and STG (ROI7 and ROI-14) (Table 3). In addition, whole brain connectivity maps revealed that hyperconnectivity was found between left SMG and medial-prefrontal cortices (mPFC) in patients with bipolar disorder (Figure 2a). On the other hand, the SMG had decreased connectivity with the left medial STG in bipolar I disorder and bipolar II disorder. The left STS (ROI-13) had increased connectivity with the bilateral posterior cingulate cortex (PCC) in bipolar I disorder and bipolar II disorder and increased connectivity with the dorsal prefrontal cortices in the schizophrenia group (Figure 2b). The left lateral superior temporal gyrus had increased connectivity with the medial STG in the bipolar I disorder and schizophrenia groups and decreased connectivity in the bipolar II disorder group (Figure 2c). 
Table 1: Demographic and clinical variables of the participants

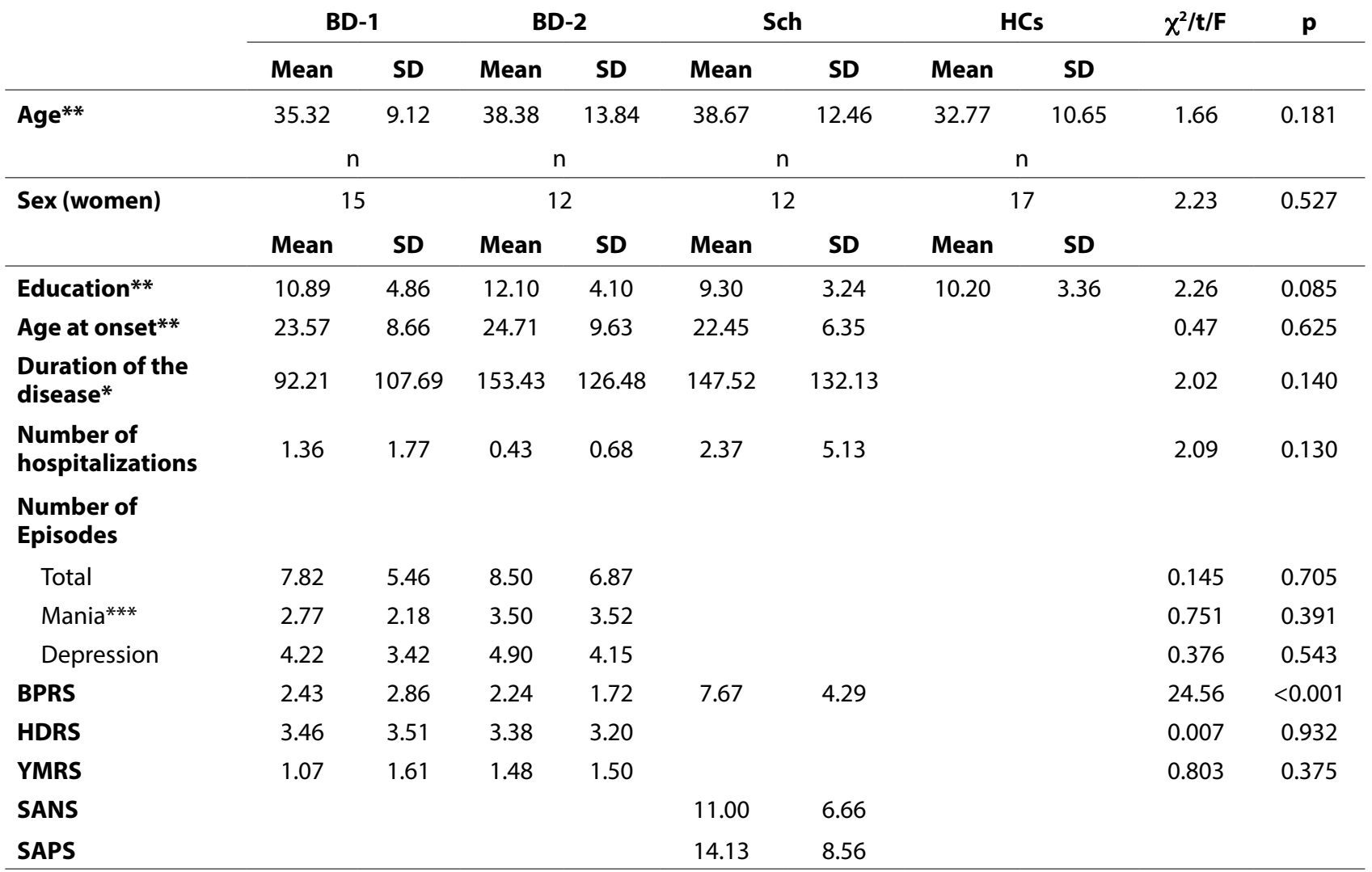

One-way ANOVA, SD, Chi-square and t tests. BD-1: Bipolar 1 Disorder, BD-2: Bipolar 2 Disorder, Sch: Schizophrenia, HCs: Healthy Controls. BPRS: Brief Psychiatric Rating Scale, HDRS: Hamilton Depression Rating Scale, YMRS: Young Mania Rating Scale, SANS: Scale for the Assessment of Negative Symptoms, SAPS: Scale for the Assessment of Positive Symptoms, *Months, ${ }^{* *}$ Years, ${ }^{* * *}$ Hypomania for BD-2

Table 2: Regions with significant volume changes across groups. Significant ROls are shown highlighted in yellow and marginally significant ones in orange

\begin{tabular}{|c|c|c|c|c|}
\hline ROls & HS & Regions & p-Value & Post-hoc Comparisons \\
\hline 1 & Right & Angular Gyrus (AG) & 0.3960 & - \\
\hline 2 & Right & Supramarginal Gyrus (SMG) & 0.5104 & - \\
\hline 3 & Right & Precentral sulcus (PCS) & 0.2896 & - \\
\hline 4 & Right & Frontal operculum (FO) & 0.1814 & - \\
\hline 5 & Right & Lateral superior temporal gyrus (L-STG) & 0.0578 & $\begin{array}{l}\mathrm{HC}>\mathrm{Sch}(p=0.0117) \\
\mathrm{HC}>\mathrm{BD}-1(p=0.0610)\end{array}$ \\
\hline 6 & Right & Superior temporal sulcus (STS) & 0.1388 & - \\
\hline 7 & Right & Superior temporal gyrus (STG) & 0.5903 & - \\
\hline 8 & Left & Angular Gyrus & 0.0223 & $\begin{array}{l}\mathrm{HC}>\mathrm{Sch}(\mathrm{p}=0.0246) \\
\mathrm{HC}>\mathrm{BD}-2(p=0.0082)\end{array}$ \\
\hline 9 & Left & Supramarginal Gyrus & 0.3976 & - \\
\hline 10 & Left & Frontal operculum & 0.0557 & - \\
\hline 11 & Left & Frontal operculum & 0.0822 & - \\
\hline 12 & Left & Lateral superior temporal gyrus & 0.0920 & - \\
\hline 13 & Left & Superior temporal sulcus & 0.0680 & - \\
\hline 14 & Left & Superior temporal gyrus & 0.0185 & $\mathrm{HC}>\mathrm{Sch}(p=0.0027)$ \\
\hline
\end{tabular}

HC: Healthy Controls, Sch: Schizophrenia, BD-1: Bipolar 1 Disorder, BD-2: Bipolar 2 Disorder 
Table 3: Pairwise connectivity differences across groups are shown as mean and standard error plots in the "Groups" column, post-hoc tests shown where pairwise group comparisons have significant differences

ROIs

Groups

p

Post-Hoc

ROI-2 - ROI-9

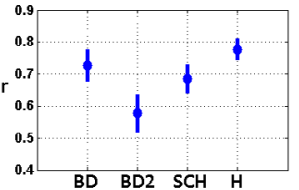

0.0091

BD-1>BD-2 $(p=0.060)$

$\mathrm{HCs}>\mathrm{BD}-2(\mathrm{p}=0.0052)$

ROI-2 - ROI-14

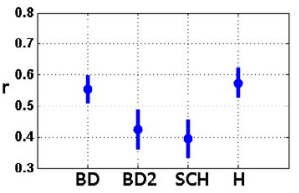

$\mathrm{HCs}>\mathrm{Sch}(\mathrm{p}=0.035)$

0.0170

BD-1 $>\operatorname{Sch}(p=0.080)$

ROI-6 - ROI-12

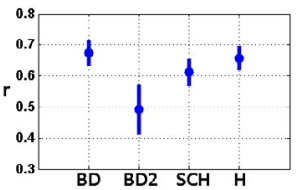

0.0369

BD-1 $>B D-2(p=0.0395)$

$\mathrm{HCs}>\mathrm{BD}-2(\mathrm{p}=0.055)$

ROI-8 - ROI-13

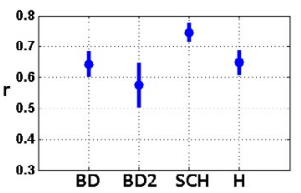

0.0372

Sch $>$ BD-2 $(p=0.0244)$

ROI-7 - ROI-14

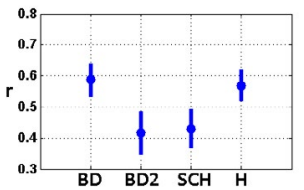

0.0427

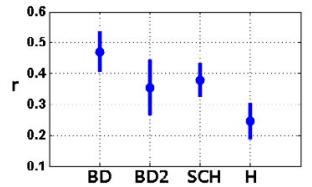

0.0491

$B D-2>H C s(p=0.0359)$

ROI-3 - ROI-5

\section{DISCUSSION}

In this study, the most significant volume difference between the groups was in the superior temporal cortices. The gray matter volume was smaller in the left hemisphere AG, lateral and medial STG in patients with schizophrenia. Interestingly, there was a difference between the bipolar disorder subgroups: the effect on the angular gyrus was only significant in bipolar I disorder, and similarly the lateral STG (Wernicke's
Area) was only affected significantly in bipolar I disorder. Connectivity differences were similar between bipolar I disorder and bipolar II disorder groups. There was increased connectivity between the left SMG mPFC and the left STS - PCC in the bipolar disorder subgroups. These groups had decreased connectivity between the left lateral STG and the bilateral medial STG. Decreased functional connectivity observed bilaterally in precentral gyrus and inferior parietal lobes was only seen in the bipolar II disorder group (Table 3, Figure 2). The schizophrenia group had increased 
connectivity between left superior temporal sulcus and bilateral dorsal prefrontal cortices.

A connectivity change between lateral and medial STG in bipolar disorder might indicate a deficit in the integration of processed auditory information from specialized tonotopic fields of the primary auditory cortices, which may lead to impaired auditory information processing. Ambiguity of auditory information may lead to deficits in cognitive or emotional auditory information processing. Abnormal activation and connectivity changes in components of the auditory networks may be related with several cognitive mechanisms. Deficient cortical auditory processing, emotional motivation, beliefs, cognitive bias, intentional alteration of attention to auditory inputs and insight might be related with auditory cortex abnormalities $(43,44)$. In addition, the mPFCs were associated with selective attentional and executive networks (45), and hyper-connectivity between the left STG and MPFC may reflect increased coupling in higher cognitive networks for auditory processing. The mPFC and PCC are components of the default mode network, and dysconnectivity in this network may reflect self-referential thought disorders (46). These findings are in line with a hypothesis postulating that dysfunction of association areas might play an important role in cognitive decline in schizophrenia and bipolar disorder (47). Since the auditory system is related to various symptoms and cognitive dysfunction in schizophrenia and bipolar disorder (2), dysconnectivity between the auditory cortices might be related with the symptoms and deficits involved. This study was performed in resting state; task-based studies may investigate the association more reliably. On the other hand, the symptoms are perpetual and are observed even at rest. Therefore, resting-state activity may also reflect disturbances in spontaneous activity.

A substantial body of evidence has demonstrated volumetric decay in gray matter (14), cortical folding abnormalities (48-50) and deteriorated laminar structure (3) in the superior temporal cortices in schizophrenia. Moreover, structural alterations $(51,52)$, volumetric loss (15), metabolic abnormalities $(53,54)$ and functional alterations $(13,23,47,55,56)$ of the auditory cortices were related to auditory hallucinations in patients with schizophrenia. Enhanced resting-state activity of the primary auditory cortices and auditory hallucinations were predisposed by aberrant connectivity between the default-mode network and auditory cortices. While the schizophrenia patients were not categorized according to hallucinations, the whole group had abnormal connectivity in both schizophrenia and bipolar disorder groups.
Volumetric loss in bipolar I disorder was observed in the STG, and this finding is in line with a metaanalysis in bipolar disorder (57). Moreover, auditory processing abnormalities $(6,7,11,12,16)$ are more severe in psychotic patients with bipolar disorder than in nonpsychotic patients (11). The bipolar II disorder group had a significant volumetric decrease in the AG. The AG region has been evaluated as a physiologic trait marker of depression, mania and euthymia in bipolar II disorder (58). The bipolar II disorder group had decreased connectivity between Wernicke's Area and the primary auditory cortices, while this finding was not observed in bipolar I disorder.

Limitations of this study are its small sample size; some of our results showed trend level significance and thus should be tested further in larger samples. The cross-sectional nature of our study did not allow us to perform analyses for clinical factors and medications. In addition, longer functional scan times are preferable to understand the attentional alterations better. Clinical variables such as medications of psychosis (for bipolar disorder) could not be controlled.

Overall, the schizophrenia group was more prone to displaying volumetric changes in the auditory cortices. On the other hand, functional alterations were observed in the bipolar disorder groups. These findings suggest that differences in the functional connectivity of restingstate networks in bipolar disorder and schizophrenia are related with functional auditory connections. Development of the auditory cortices continues until late adolescence, and neurobiological effects of schizophrenia and bipolar disorder may disrupt the development of the auditory networks (1). Impairment of the auditory networks may predispose for either symptoms or cognitive dysfunction. The accumulation of further evidence may help to determine abnormalities in networks for specific disorders. Further studies considering symptoms and cognitive background of these disorders may contribute to our understanding of the neurobiology of psychiatric conditions.

\begin{tabular}{|c|c|c|}
\hline \multicolumn{2}{|c|}{ Contribution Categories } & \multirow{2}{*}{$\begin{array}{l}\text { Author Initials } \\
\text { M.I.A., D.O., M.P., C.M. }\end{array}$} \\
\hline \multirow{3}{*}{ Category 1} & Concept/Design & \\
\hline & Data acquisition & M.I.A., S.S.C., S.U.K., A.C. \\
\hline & Data analysis/Interpretation & M.I.A., B.A., O.A., E.M.S. \\
\hline \multirow{2}{*}{ Category 2} & Drafting manuscript & M.I.A., B.A., E.M.S. \\
\hline & Critical revision of manuscript & C.M., M.P., E.M.S \\
\hline Category 3 & Final approval and accountability & $\begin{array}{l}\text { M.I.A., B.A., O.A., E.M.S., } \\
\text { S.S.C., S.U.K., A.C., C.M., } \\
\text { M.P., D.O. }\end{array}$ \\
\hline \multirow{3}{*}{ Other } & Technical or material support & B.A., O.A., E.M.S. \\
\hline & Supervision & M.P., C.M., D.O. \\
\hline & Securing funding (if applicable) & N/A \\
\hline
\end{tabular}


Ethics Committee Approval: The study was approved by the Local Ethics Committee.

Informed Consent: Written informed consent was obtained from the patient for the publication of the case report and the accompanying images.

Peer-review: Externally peer-reviewed.

Conflict of Interest: Dr. Kaymak participated in a relevant Otsuka Pharmaceutical clinical trial. Dr. Can participated in a relevant Janssen Pharmaceutica clinical trial. The other authors declare that they have no conflicts of interest.

Financial Disclosure: The study was funded by the Scientific Research Commission of Ankara Yildirim Beyazit University (Project No. 803).

\section{REFERENCES}

1. Moore DR. Auditory development and the role of experience. $\mathrm{Br}$ Med Bull 2002; 63:171-181.

2. Bora E, Yucel M, Pantelis C. Cognitive endophenotypes of bipolar disorder: a meta-analysis of neuropsychological deficits in euthymic patients and their first-degree relatives. J Affect Disord 2009; 113:1-20.

3. Chance SA, Casanova MF, Switala AE, Crow TJ. Auditory cortex asymmetry, altered minicolumn spacing and absence of ageing effects in schizophrenia. Brain 2008; 131:3178-192.

4. McNamara RK, Rider T, Jandacek R, Tso P. Abnormal fatty acid pattern in the superior temporal gyrus distinguishes bipolar disorder from major depression and schizophrenia and resembles multiple sclerosis. Psychiatry Res 2014; 215:560567.

5. Nudmamud S, Reynolds LM, Reynolds GP. N-acetylaspartate and $\mathrm{N}$-Acetylaspartylglutamate deficits in superior temporal cortex in schizophrenia and bipolar disorder: a postmortem study. Biol Psychiatry 2003; 53:1138-1141.

6. Atagun MI, Guntekin B, Ozerdem A, Tulay E, Basar E. Decrease of theta response in euthymic bipolar patients during an oddball paradigm. Cogn Neurodyn 2013; 7:213-223.

7. Atagun M, Guntekin B, Masali B, Tulay E, Basar E. Decrease of event-related delta oscillations in euthymic patients with bipolar disorder. Psychiatry Res 2014; 223:43-48.

8. Atagun MI, Sikoglu EM, Can SS, Karakas-Ugurlu G, UlusoyKaymak S, Caykoylu A, Algin O, Phillips ML, Moore CM, Ongur D. Investigation of Heschl's gyrus and planum temporale in patients with schizophrenia and bipolar disorder: a proton magnetic resonance spectroscopy study. Schizophr Res 2015; 161:202-209.

9. Atagun MI, Sikoglu EM, Soykan C, Serdar Suleyman C, UlusoyKaymak S, Caykoylu A, Algin O, Phillips ML, Ongur D, Moore CM. Perisylvian GABA levels in schizophrenia and bipolar disorder. Neurosci Lett 2017; 637:70-74.

10. Atagun MI. Brain oscillations in bipolar disorder and lithiuminduced changes. Neuropsychiatr Dis Treat 2016; 12:589-601.

11. Onitsuka T, Oribe N, Kanba S. Neurophysiological findings in patients with bipolar disorder. Suppl Clin Neurophysiol 2013; 62:197-206.

12. Onitsuka T, Oribe N, Nakamura I, Kanba S. Review of neurophysiological findings in patients with schizophrenia. Psychiatry Clin Neurosci 2013; 67:461-470.

13. Alderson-Day B, McCarthy-Jones S, Fernyhough C. Hearing voices in the resting brain: a review of intrinsic functional connectivity research on auditory verbal hallucinations. Neurosci Biobehav Rev 2015; 55:78-87.

14. Vita A, De Peri L, Deste G, Sacchetti E. Progressive loss of cortical gray matter in schizophrenia: a meta-analysis and metaregression of longitudinal MRI studies. Transl Psychiatry 2012; 2:e190.

15. Jardri R, Pouchet A, Pins D, Thomas P. Cortical activations during auditory verbal hallucinations in schizophrenia: a coordinate-based meta-analysis. Am J Psychiatry 2011; 168:7381 .

16. Ozerdem A, Guntekin B, Atagun I, Turp B, Basar E. Reduced long distance gamma $(28-48 \mathrm{~Hz})$ coherence in euthymic patients with bipolar disorder. J Affect Disord 2011; 132:325332.

17. Atagun MI, Sikoglu EM, Can SS, Ugurlu GK, Kaymak SU, Caykoylu A, Algin O, Phillips ML, Moore CM, Ongur D. Neurochemical differences between bipolar disorder type I and II in superior temporal cortices: a proton magnetic resonance spectroscopy study. J Affect Disord 2018; 235:15-19.

18. Kusmierek P, Ortiz M, Rauschecker JP. Sound-identity processing in early areas of the auditory ventral stream in the macaque. J Neurophysiol 2012; 107:1123-1141.

19. Alho K, Rinne T, Herron TJ, Woods DL. Stimulus-dependent activations and attention-related modulations in the auditory cortex: a meta-analysis of fMRI studies. Hear Res 2014; 307:2941.

20. Rauschecker JP, Scott SK. Maps and streams in the auditory cortex: nonhuman primates illuminate human speech processing. Nat Neurosci 2009; 12:718-724.

21. Jardri R, Deneve S. Circular inferences in schizophrenia. Brain 2013; 136:3227-3241.

22. Kuhn S, Gallinat J. Quantitative meta-analysis on state and trait aspects of auditory verbal hallucinations in schizophrenia. Schizophr Bull 2012; 38:779-786.

23. Shinn AK, Baker JT, Cohen BM, Ongur D. Functional connectivity of left Heschl's gyrus in vulnerability to auditory hallucinations in schizophrenia. Schizophr Res 2013; 143:260268.

24. Choppin S, Trost W, Dondaine T, Millet B, Drapier D, Verin M, Robert G, Grandjean D. Alteration of complex negative emotions induced by music in euthymic patients with bipolar disorder. J Affect Disord 2016; 191:15-23.

25. Zenisek R, Thaler NS, Sutton GP, Ringdahl EN, Snyder JS, Allen DN. Auditory processing deficits in bipolar disorder with and without a history of psychotic features. Bipolar Disord 2015; 17:769-780. 
26. Du Y, Pearlson GD, Liu J, Sui J, Yu Q, He H, Castro E, Calhoun VD. A group ICA based framework for evaluating resting fMRI markers when disease categories are unclear: application to schizophrenia, bipolar, and schizoaffective disorders. Neuroimage 2015; 122:272-280.

27. Rashid B, Damaraju E, Pearlson GD, Calhoun VD. Dynamic connectivity states estimated from resting fMRI Identify differences among Schizophrenia, bipolar disorder, and healthy control subjects. Front Hum Neurosci 2014; 8:897.

28. Joel SE, Caffo BS, van Zijl PC, Pekar JJ. On the relationship between seed-based and ICA-based measures of functional connectivity. Magn Reson Med 2011; 66:644-657.

29. First MB, Spitzer RL, Gibbon M, Williams JBW. Structured Clinical Interview for DSM-IV-TR Axis I Disorders, Research Version, Patient Edition. (SCID-I/P). New York, USA: Biometric Research, New York State Psychiatric Institute; 2002.

30. Young RC, Biggs JT, Ziegler VE, Meyer DA. A rating scale for mania: reliability, validity and sensitivity. Br J Psychiatry 1978; 133:429-435.

31. Karadag F, Oral ET, Yalcin F, Erten E. Reliability and validity of Turkish translation of Young Mania Rating Scale. Turk Psikiyatri Derg 2002; 13:107-114.

32. Hamilton M. A rating scale for depression. J Neurol Neurosurg Psychiatry 1960; 23:56-62.

33. Akdemir A, Orsel SD, Dag I, Turkcapar MH, Iscan N, Ozbay H. Clinical use and the reliability and validity of the Turkish version of the Hamilton Depression Rating Scale (HDRS). Psychiatry Psychology and Psychopharmacology 1996; 4:251-259. (Turkish)

34. Overall JE, Gorham DR. The Brief Psychiatric Rating Scale. Psychological Reports 1962; 10:799-812.

35. Andreasen NC. The Scale for the Assessment of Negative Symptoms (SANS): conceptual and theoretical foundations. Br J Psychiatry Suppl 1989; 7:49-58.

36. Erkoc S, Arkonac O, Atakli C, Ozmen E. The reliability and validity of scale for the assesment of the negative symstoms. Dusunen Adam The Journal of Psychiatry and Neurological Sciences 1991; 4:14-15. (Turkish)

37. Andreasen NC. The scale for the assessment of Positive Symptoms (SAPS). Iowa, USA: The University of Iowa; 1984.

38. Erkoc S, Arkonac O, Atakli C, Ozmen E. The reliability and validit of scale for the assesment of the Positive Symtoms. Dusunen Adam The Journal of Psychiatry and Neurological Sciences 1991; 4:20-24. (Turkish)

39. Oldfield RC. The assessment and analysis of handedness: the Edinburgh inventory. Neuropsychologia 1971; 9:97-113.

40. Fischl B, van der Kouwe A, Destrieux C, Halgren E, Segonne F, Salat DH, Busa E, Seidman LJ, Goldstein J, Kennedy D, Caviness V, Makris N, Rosen B, Dale AM. Automatically parcellating the human cerebral cortex. Cereb Cortex 2004; 14:11-22.

41. McKeown MJ, Hansen LK, Sejnowsk TJ. Independent component analysis of functional MRI: what is signal and what is noise? Curr Opin Neurobiol 2003; 13:620-629.
42. Fox MD, Snyder AZ, Vincent JL, Corbetta M, Van Essen DC, Raichle ME. The human brain is intrinsically organized into dynamic, anticorrelated functional networks. Proc Natl Acad Sci USA 2005; 102:9673-9678.

43. Waters F, Allen P, Aleman A, Fernyhough C, Woodward TS, Badcock JC, Barkus E, Johns L, Varese F, Menon M, Vercammen A, Larøi F. Auditory hallucinations in schizophrenia and nonschizophrenia populations: a review and integrated model of cognitive mechanisms. Schizophr Bull 2012; 38:683-693

44. Waters F, Fernyhough C. Hallucinations: a systematic review of points of similarity and difference across diagnostic classes. Schizophr Bull 2017; 43:32-43.

45. Jimenez AM, Lee J, Green MF, Wynn JK. Functional connectivity when detecting rare visual targets in schizophrenia. Psychiatry Res Neuroimaging 2017; 261:35-43.

46. Marchand WR, Lee JN, Johnson S, Gale P, Thatcher J. Differences in functional connectivity in major depression versus bipolar II depression. J Affect Disord 2013; 150:527-532.

47. Baker JT, Holmes AJ, Masters GA, Yeo BT, Krienen F, Buckner $\mathrm{RL}$, Ongur D. Disruption of cortical association networks in schizophrenia and psychotic bipolar disorder. JAMA Psychiatry 2014; 71:109-118.

48. Cachia A, Paillere-Martinot ML, Galinowski A, Januel D, de Beaurepaire R, Bellivier F, Artiges E, Andoh J, Bartrés-Faz D, Duchesnay E, Rivière D, Plaze M, Mangin JF, Martinot JL. Cortical folding abnormalities in schizophrenia patients with resistant auditory hallucinations. Neuroimage 2008; 39:927935.

49. Penttila J, Paillere-Martinot ML, Martinot JL, Ringuenet D, Wessa M, Houenou J, Gallarda T, Bellivier F, Galinowski A, Bruguière P, Pinabel F, Leboyer M, Olié JP, Duchesnay E, Artiges E, Mangin JF, Cachia A. Cortical folding in patients with bipolar disorder or unipolar depression. J Psychiatry Neurosci 2009; 34:127-135.

50. Penttila J, Paillere-Martinot ML, Martinot JL, Mangin JF, Burke L, Corrigall R, Frangou S, Cachia A. Global and temporal cortical folding in patients with early-onset schizophrenia. J Am Acad Child Adolesc Psychiatry 2008; 47:1125-1132.

51. Hubl D, Koenig T, Strik W, Federspiel A, Kreis R, Boesch C, Maier SE, Schroth G, Lovblad K, Dierks T. Pathways that make voices: white matter changes in auditory hallucinations. Arch Gen Psychiatry 2004; 61:658-668.

52. Seok JH, Park HJ, Chun JW, Lee SK, Cho HS, Kwon JS, Kim JJ. White matter abnormalities associated with auditory hallucinations in schizophrenia: a combined study of voxel-based analyses of diffusion tensor imaging and structural magnetic resonance imaging. Psychiatry Res 2007; 156:93-104.

53. Homan P, Vermathen P, Van Swam C, Federspiel A, Boesch C, Strik W, Dierks T, Hubl D, Kreis R. Magnetic resonance spectroscopy investigations of functionally defined language areas in schizophrenia patients with and without auditory hallucinations. Neuroimage 2014; 94:23-32.

54. Hugdahl K, Craven AR, Nygard M, Loberg EM, Berle JO, Johnsen E, Kroken R, Specht K, Andreassen OA, Ersland L. 
Glutamate as a mediating transmitter for auditory hallucinations in schizophrenia: a (1)H MRS study. Schizophr Res 2015; $161: 252-260$

55. Lefebvre S, Demeulemeester M, Leroy A, Delmaire C, Lopes R, Pins D, Thomas P, Jardri R. Network dynamics during the different stages of hallucinations in schizophrenia. Human Brain Mapp 2016; 37:2571-2586.

56. Chahine G, Richter A, Wolter S, Goya-Maldonado R, Gruber O. Disruptions in the left frontoparietal network underlie resting state endophenotypic markers in schizophrenia. Hum Brain Mapp 2017; 38:1741-1750.

57. Hanford LC, Nazarov A, Hall GB, Sassi RB. Cortical thickness in bipolar disorder: a systematic review. Bipolar Disord 2016; 18:4-18.

58. Vizueta N, Rudie JD, Townsend JD, Torrisi S, Moody TD, Bookheimer SY, Altshuler LL. Regional fMRI hypoactivation and altered functional connectivity during emotion processing in nonmedicated depressed patients with bipolar II disorder. Am J Psychiatry 2012; 169:831-840. 